University of Wollongong

Research Online

Faculty of Informatics - Papers (Archive)

Faculty of Engineering and Information

Sciences

$1-1-2009$

\title{
A simulation study on the energy efficiency of pure and slotted Aloha based RFID tag reading protocols
}

\author{
Alejandro Ruiz-Rivera \\ University of Wollongong \\ Dheeraj Klair \\ University of Wollongong, dkk282@uow.edu.au \\ Kwan-Wu Chin \\ University of Wollongong, kwanwu@uow.edu.au
}

Follow this and additional works at: https://ro.uow.edu.au/infopapers

Part of the Physical Sciences and Mathematics Commons

\section{Recommended Citation}

Ruiz-Rivera, Alejandro; Klair, Dheeraj; and Chin, Kwan-Wu: A simulation study on the energy efficiency of pure and slotted Aloha based RFID tag reading protocols 2009, 1-5.

https://ro.uow.edu.au/infopapers/1408

Research Online is the open access institutional repository for the University of Wollongong. For further information contact the UOW Library: research-pubs@uow.edu.au 


\title{
A simulation study on the energy efficiency of pure and slotted Aloha based RFID tag reading protocols
}

\author{
Abstract \\ This paper studies the energy efficiency of twelve Pure and Slotted Aloha tag reading protocol variants via \\ simulation. We compare their energy consumption in three collision resolution phases: 1) success, 2) \\ collision, and 3 ) idle. Our extensive simulation results show that Pure Aloha with fast mode and muting \\ has the lowest energy consumption, and hence is most suited for deployment in energy-constrained \\ environments.

\section{Keywords} \\ simulation, study, energy, efficiency, pure, slotted, Aloha, based, RFID, tag, reading, protocols

\section{Disciplines} \\ Physical Sciences and Mathematics

\section{Publication Details} \\ A. Ruiz-Rivera, D. Klair \& K. Chin, "A simulation study on the energy efficiency of pure and slotted Aloha \\ based RFID tag reading protocols," in 2009 6th IEEE Consumer Communications and Networking \\ Conference, 2009, pp. 1-5.
}




\title{
A Simulation Study on the Energy Efficiency of Pure and Slotted Aloha based RFID Tag Reading Protocols
}

\author{
Alejandro Ruiz R., Dheeraj K. Klair and Kwan-Wu Chin \\ School of Electrical, Computer, and Telecommunications Engineering \\ University of Wollongong \\ Northfields Avenue, NSW, Australia \\ \{arr506, dkk282, kwanwu\}@uow.edu.au
}

\begin{abstract}
This paper studies the energy efficiency of twelve Pure and Slotted Aloha tag reading protocol variants via simulation. We compare their energy consumption in three collision resolution phases: 1) success, 2) collision, and 3) idle. Our extensive simulation results show that Pure Aloha with fast mode and muting has the lowest energy consumption, and hence is most suited for deployment in energy-constrained environments.
\end{abstract}

Index Terms-RFID, anti-collision, tag reading, energy consumption, Aloha

\section{INTRODUCTION}

Radio Frequency IDentification (RFID) technologies are emerging to be a favorite alternative to bar-codes due to their non line of sight object identification capabilities [13]. In addition, RFID tags are available in a variety of shapes, sizes, form factors and ranges, thereby making them suitable for applications in crowd control, e-passports, etc [2].

Recently, researchers have proposed RFID-enhanced wireless sensor networks (WSNs) [5][3], where sensor nodes are equipped with an RFID reader. The resulting network can then be used to instrument a physical space in order to track RFID tagged objects; e.g., in a library to track books. Their key limitation, however, is the amount of energy that a RFID reader spends reading or scanning RFID tags. In this respect, apart from our earlier analytical work [6], no other works have performed extensive simulation studies on Aloha based tag reading protocols. Though studies on the energy efficiency of tree based tag reading protocols exist [8], they are unsuitable for use in RFID-enhanced WSNs due to their excessive complexity, significant memory overheads, and complex hardware. In comparison, Aloha protocols promise lower bandwidth, lower reader to tag transmissions, dynamic adaptability to varying loads, simpler reader designs, and lower memory requirements.

Henceforth, this paper investigates, via simulation, the performance of Pure Aloha and Slotted Aloha tag reading protocols, and also their variants. We compare their energy consumption in the following phases of the anti-collision phases: 1) success, 2) collision, and 3) idle listening. From our extensive simulation studies, we found Pure Aloha fast mode variants to have the best performance. These variants and specifically Pure Aloha with fast mode and muting showed the smallest average number of collisions, hence the highest number of successes. As a result, an RFID enhanced sensor node using this variant is expected to expend the least amount of energy when reading tags. Note, in our earlier work [6], besides our analytical results, we only considered six protocols. In this paper, we investigate twelve Aloha variants, especially those using the fast mode and slow down feature.

This paper is organized as follows. Section II introduces the tag collision problem, and tag reading protocols of interest. Following that, in Section III, we present our simulation research methodology. Lastly, Section IV and V present our results and conclusions respectively.

\section{BACKGROUND}

A key problem in RFID systems is collision, where multiple tags within the reader's field reply at the same time [2]; see Figure 1. This problem causes bandwidth and energy wastage, leading to increased identification delays [6][7].

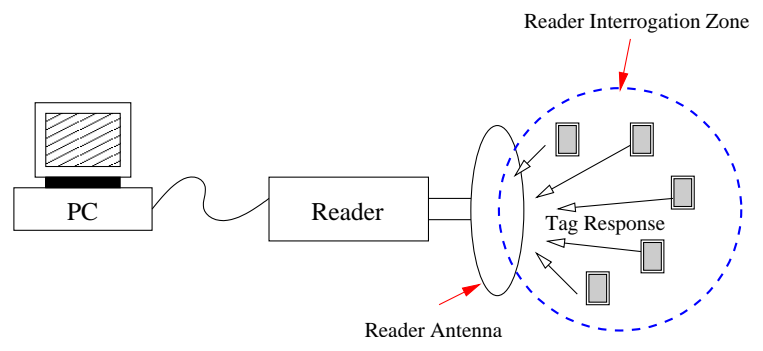

Fig. 1. Reader and tags interactions.

To address the tag collision problem, researchers have developed numerous anti-collision protocols [10] [14][1]][4]. The simplest ones are based on Pure and Slotted Aloha [14]. In Pure Aloha based RFID systems, once a tag has been energized by a reader's field, it responds at random times. The tag then waits for the reader to respond, which can either be a positive acknowledgement $(A C K)$ if the reader receives the tag's ID correctly, or a negative acknowledgement $(N A C K)$, meaning a collision has occurred and the tag is required to retransmit after a random time.

Pure Aloha based tag reading protocols, however, suffer from the well known partial collision problem that bounds 
their throughput to $18 \%$ [9]. This motivated researchers to propose the following improvements:

- Pure Aloha with Muting. When muting is used, the number of tags in a reader's interrogation zone is reduced after each successful tag response, meaning the offered load to the reader is reduced after a tag is identified. Figure 2 shows the behavior of Pure Aloha with muting. Initially, tags 1 and 3's transmission collide, causing them to wait a random amount of time before retransmitting again. After identification, the reader silences the tags using the "mute" command.

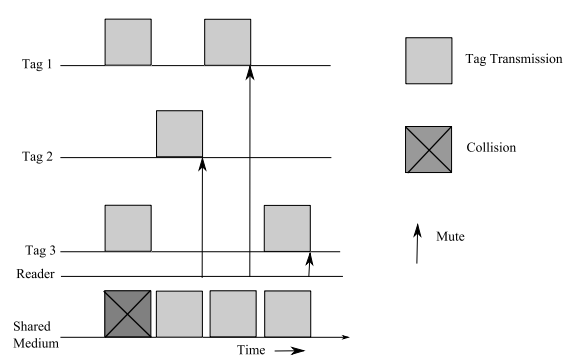

Fig. 2. Pure Aloha with muting.

- Pure Aloha with Slow Down. Instead of being muted, a tag can be instructed using a "slow down" command to reduce its rate of transmission, hence decreasing the probability of collision. Figure 3 shows how the reader slows tag 1 down after identification, where tag 1 adapts its random back-off counter to decrease its transmission rate.

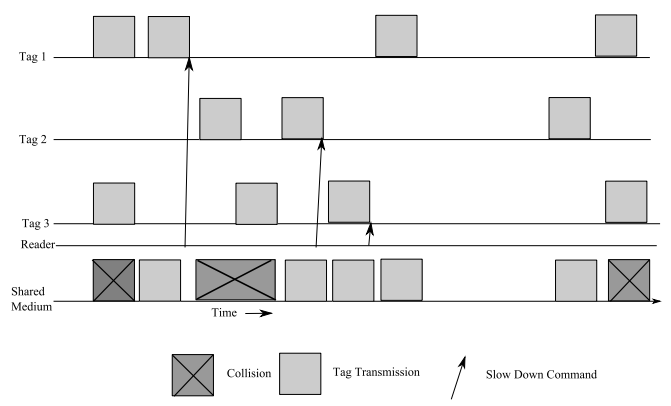

Fig. 3. Pure Aloha with slow down.

- Pure Aloha with Fast Mode. A "silence" command is sent by the reader once it has detected the start of a tag transmission. This command has the effect of stopping other tags from transmitting. Tags are allowed to transmit again after the reader has sent an ACK command or until their waiting timer expires. Figure 4 shows Pure Aloha with fast mode. Once the reader detects a transmission from tag 2, tag 1 and tag 3 are silenced and reactivated only after tag 2 has finished transmitting.

- Other Variants. Lastly, we can create two more variants, namely Pure Aloha with fast mode and muting, and Pure Aloha with fast mode and slow down by combining the respective features. These variants are shown in Figures 5 and 6 respectively, where tags 1 and 3 are silenced when tag 2 starts transmitting. After tag 2 is identified, it

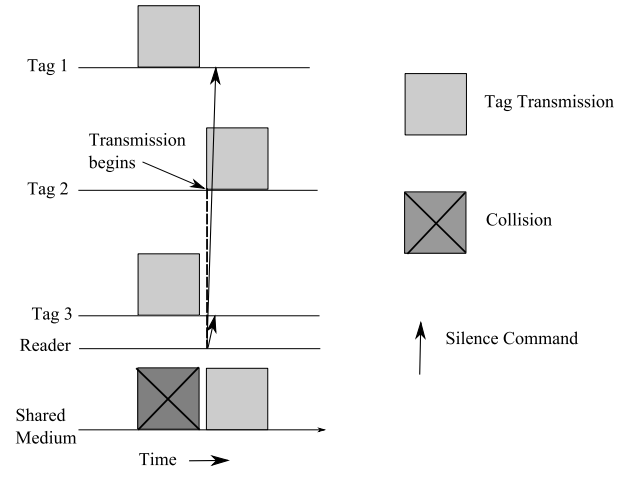

Fig. 4. Pure Aloha with fast mode.

is muted. Similarly, in Figure 6, after tag 2 is identified using fast mode, it is slowed down to allow other tags to transmit.

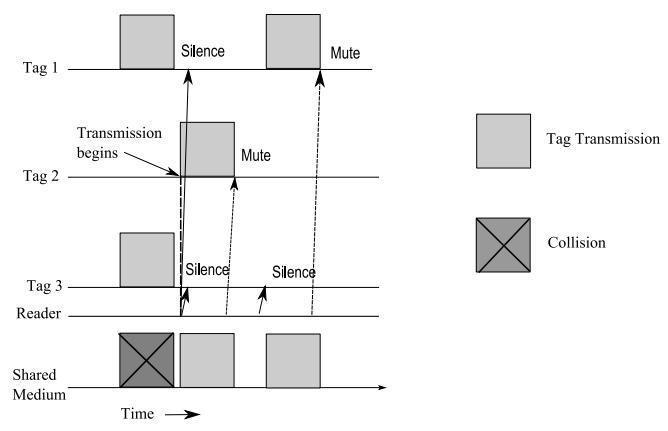

Fig. 5. Pure Aloha with fast mode and muting.

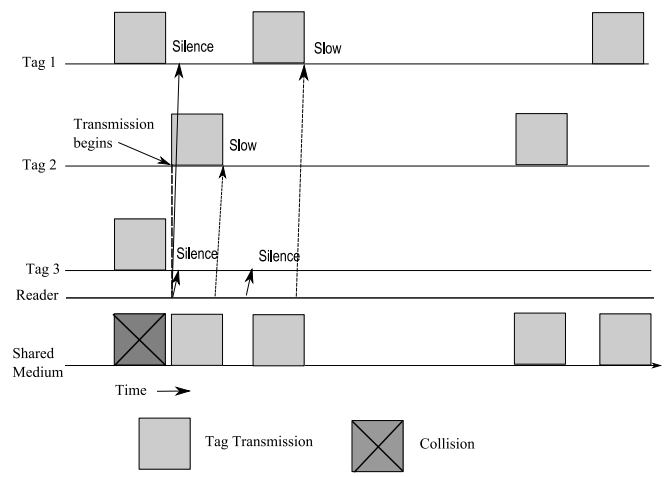

Fig. 6. Pure Aloha with fast mode and slow down.

Tag reading protocols can also use Slotted Aloha, where tags respond at pre-defined synchronous points in time. If there is a collision, tags wait for a random number of slots before retransmitting. Similar to Pure Aloha, there are various variants:

1) Slotted Aloha with Muting/Slow Down. The principle operation is similar to Pure Aloha with muting/slow down, but operates in a slotted manner.

2) Slotted Aloha with Early End. If no transmission is detected at the beginning of a slot, the reader closes the slot early. Two commands are used: start-of-frame (SOF) and end-of-frame (EOF). The former is used to 
start a reading cycle, and the later is used by the reader to close an idle slot early. Figure 5 depicts how early end is used to terminate idle slots.

3) Slotted Aloha with Early End and Muting. When tags have been identified, the reader sends a muting command, thereby reducing the number of responding tags. When no replies are detected after a small period of time, the reader closes the slot early using the EOF command.

4) Slotted Aloha with Slow Down and Early End: This combines slow down with early end feature.

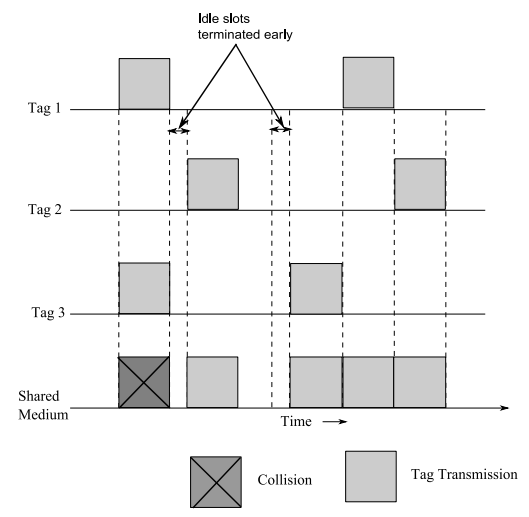

Fig. 7. Slotted Aloha with early end.

\section{A. Summary}

In summary, there are four key features being used to increase the performance of Pure and Slotted Aloha based tag reading protocols: i) muting, ii) slow down, iii) early-end, and iv) fast mode. To recap, fast mode is only used in conjunction with Pure Aloha variants to reduce their vulnerability period. Early end is used by slotted Aloha variants to reduce idle listening where idle slots are terminated early. Lastly, muting and slow down have the effect of reducing the offered load to the reader.

Given the number of available variants and our motivation to equip sensor nodes with a RFID reader, a key research question is determining variants that consume the least amount of energy. Specifically, we want to employ the variant that experiences the lowest number of collisions, minimizes reader idle time, and maximizes the number of read successes. In the next section, we first describe our simulation methodology before presenting our results in Section IV.

\section{Simulation Methodology}

We used Matlab 7.0.4 [12] to study the aforementioned protocols. We simulated an RFID-enhanced sensor node equipped with a SkyeTek M1-Mini RFID reader [11]. Each node has a Lithium rechargeable battery (B) with 480 joules of energy. The data rate from a tag to the reader is $26 \mathrm{kbps}$, as per ISO 15693 [11]. The reader consumes 180 milli-watts and 150 micro-watts during scanning and sleeping respectively.

We assume negligible processing and propagation delays. Moreover, we assume a noise free channel; i.e., packet loss are due to collisions only. Tag ID is 96 bits in length. Tags are assumed to be passive and used read-only mode. In addition, we assume tags' antenna is never at 90 degrees with respect to the reader, otherwise tags become unreadable and hence does not contribute to the offer load [2].

We investigate tag densities ranging from 1 to 150 . Each simulation is repeated 100 times, and we record the energy consumed by the reader in three phases: 1) success - the average energy consumed to read $n$ tags successfully, 2) collision - the average energy wasted in collisions to read $n$ tags, and 3) idle-listening - energy consumed while the reader waits for a response.

\section{RESUlTS}

We first compare the performance of Pure and Slotted Aloha variants separately before analyzing their performance collectively.

\section{A. Pure Aloha Variants}

Figure 10 plots the energy consumed in collisions versus the number of tags. Pure Aloha (PA) consumes the highest energy compared to its variants. Equipping Pure Aloha with slow down $(\mathrm{PA}+\mathrm{SD})$ results in fewer collisions, resulting in lower energy consumption compared to Pure Aloha. This is because fewer tags are trying to access the channel after each successful read.

Pure Aloha with muting $(\mathrm{PA}+\mathrm{M})$ shows better energy savings compared to Pure Aloha and Pure Aloha with slow down. In Pure Aloha with muting, fewer collisions are observed, given the decreasing tag numbers with each successful read.

Fast mode variants consume the lowest energy compared to other Pure Aloha variants. Once a tag starts transmitting, the reader sends a silence command to inhibit other tags from transmitting. Pure Aloha with fast mode and muting $(\mathrm{PA}+\mathrm{F}+\mathrm{M})$ combines the benefits of both features to yield the smallest number of collisions, and hence has the lowest energy wastage compared to other Pure Aloha variants.

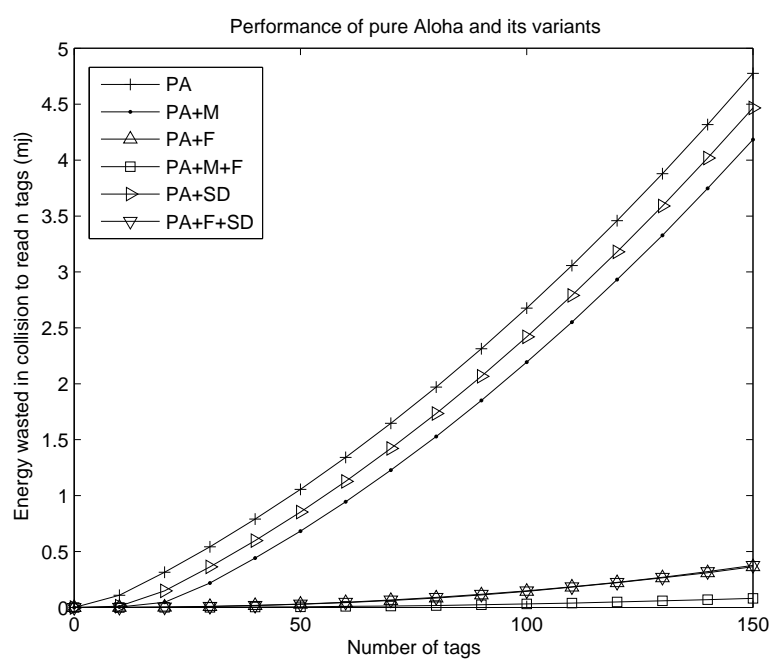

Fig. 8. Energy wasted in collisions by Pure Aloha variants

Figure 9 shows the energy wasted when a reader remains in the idle state using Pure Aloha and its variants. Pure Aloha fast 
mode variants waste more energy in idle listening compared to other Aloha variants. The key reason is the reduction in the reader's vulnerability period, which reduces collisions and increases the probability of having a free channel. Pure Aloha and its slow down/muting variants have the lowest energy wastage in idle listening because of a higher number of collisions compared to fast mode variants.

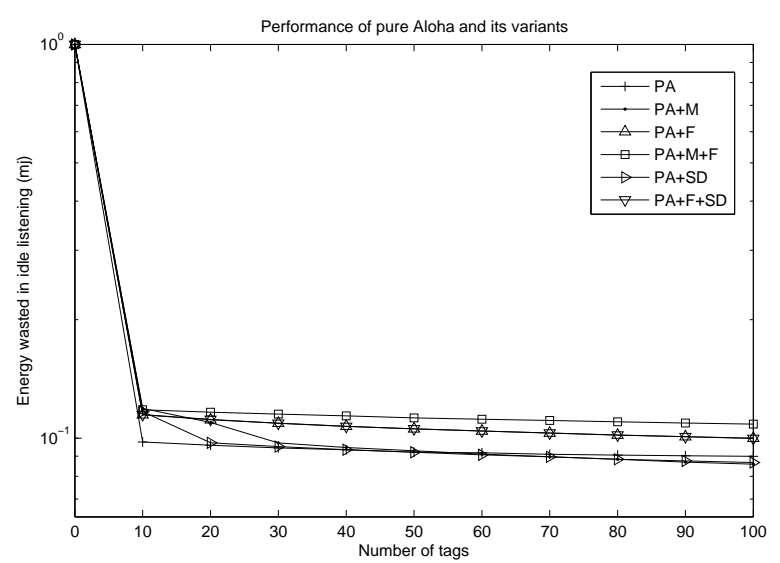

Fig. 9. Energy wasted in idle listening by Pure Aloha variants.

Figure 10 depicts the energy consumed to read $n$ tags successfully by Pure Aloha variants. Pure Aloha with fast mode and muting consumes the lowest energy among all variants. The is because of a significant drop in collisions when fast mode and muting are used together.

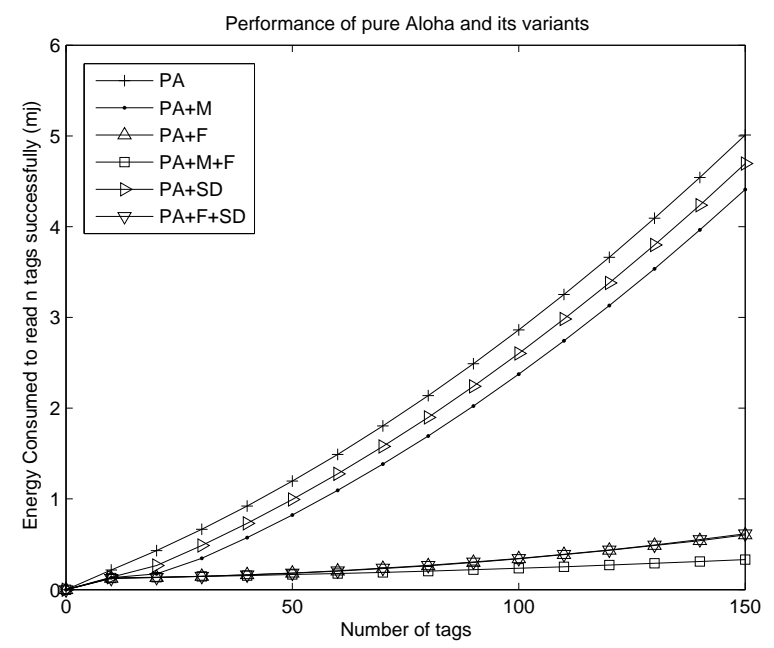

Fig. 10. Energy consumed to read $\mathrm{n}$ tags by Pure Aloha variants.

\section{B. Slotted Aloha Variants}

Figure 11 compares the energy consumption in the collision phase amongst six Slotted Aloha variants. The graph indicates that Slotted Aloha exhibits the worst performance, while Slotted Aloha with muting $(\mathrm{SA}+\mathrm{M})$ and Slotted Aloha with muting and early end $(\mathrm{SA}+\mathrm{M}+\mathrm{EE})$ demonstrate the best performance. In Slotted Aloha, tags are only allowed to transmit in fixed time slots. Therefore, the muting command decreases contention amongst tags, leading to fewer collisions. In contrast, the slow down command only has a small impact, given that it does not reduce the number of tags.

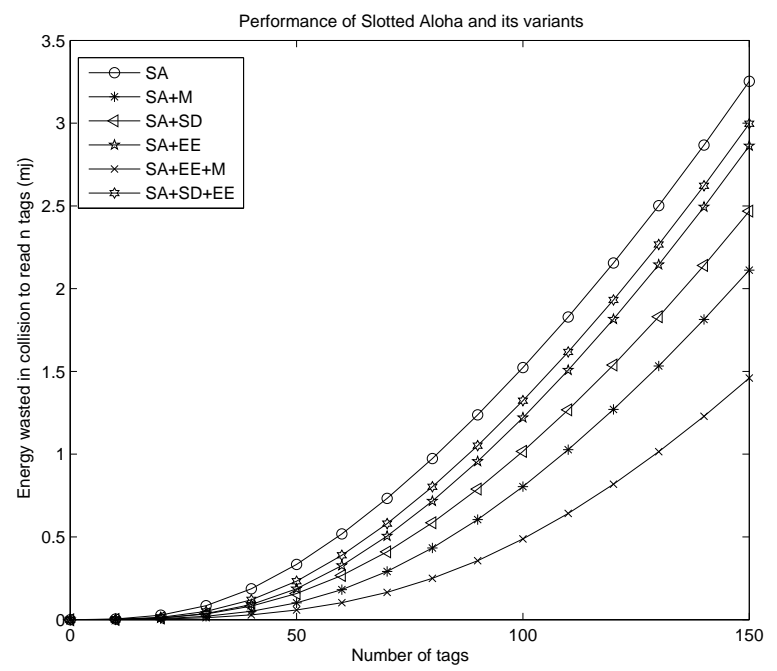

Fig. 11. Energy wasted in collisions by Slotted Aloha variants.

Figure 12 plots the energy wastage in idle listening versus the number of tags. A significant reduction in energy consumption is observed in early-end variants. This is because the reader closes idle slots early using the EOF command. Other variants such as Slotted Aloha, Slotted Aloha with muting and Slotted Aloha with slow down have a higher energy wastage in idle listening compared to those which support early end.

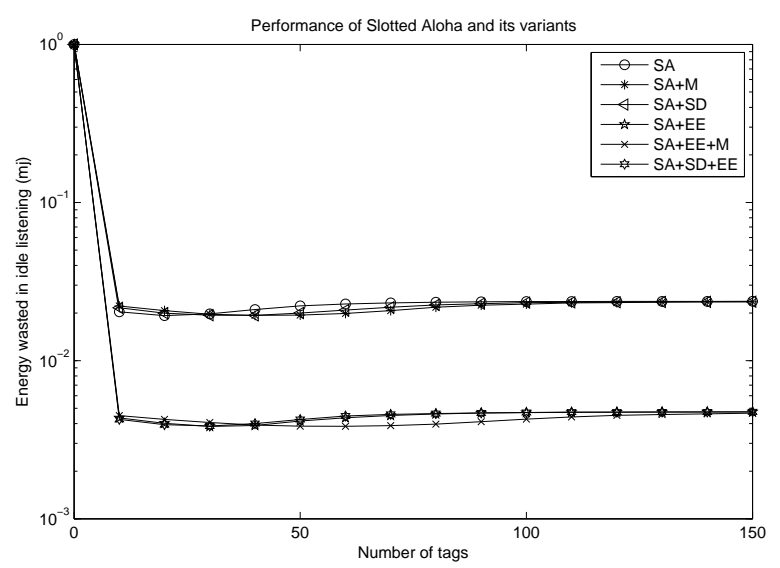

Fig. 12. Energy wasted in idle listening by Slotted Aloha variants.

Figure 13 plots the energy consumed to read $n$ tags successfully using Slotted Aloha. We can see that slotted Aloha with muting and early end consumes the lowest energy. This is because muting yields fewer collisions and early end reduces the energy expended in idle listening.

\section{All Variants}

Figure 14 plots the energy consumed in collisions by Pure and Slotted Aloha variants. In this case, Pure and Slotted 


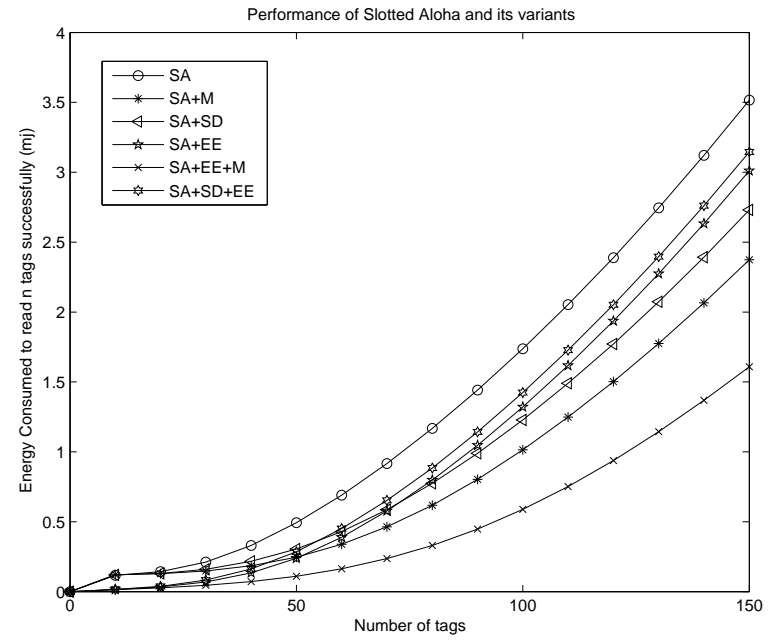

Fig. 13. Energy consumed to read $n$ tags by Slotted Aloha variants.

Aloha have the highest energy consumption amongst all variants. Pure Aloha with fast mode and muting has the best performance.

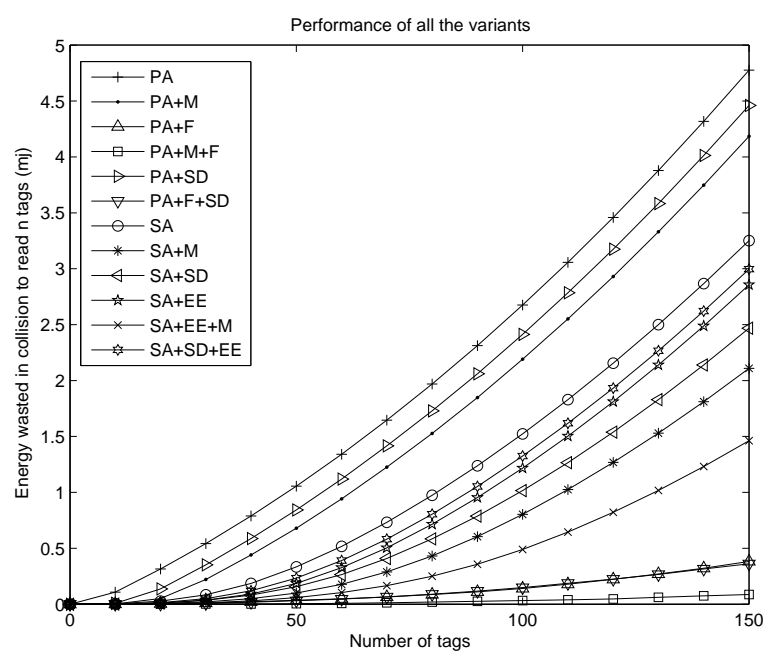

Fig. 14. Energy wasted in collisions by Pure and Slotted Aloha variants.

Figure 15 presents the reader's idle energy wastage versus the number of tags for all variants. Pure Aloha with fast mode and muting consumes the highest energy. Slotted Aloha with early end and muting has the highest energy savings in idle listening. This is because the early end feature terminates idle slots early. Variants with muting and slow down have higher energy wastage in idle listening compared to conventional Pure and Slotted Aloha. This is due to fewer collisions, which increases the probability of idle slots, and thereby extending the reader's idle time.

Figure 16 depicts the energy consumed to read a given number of tags by all Aloha variants. Pure Aloha with fast mode and its variants have the highest energy savings. Specifically, Pure Aloha with fast mode and muting consumes the lowest energy.

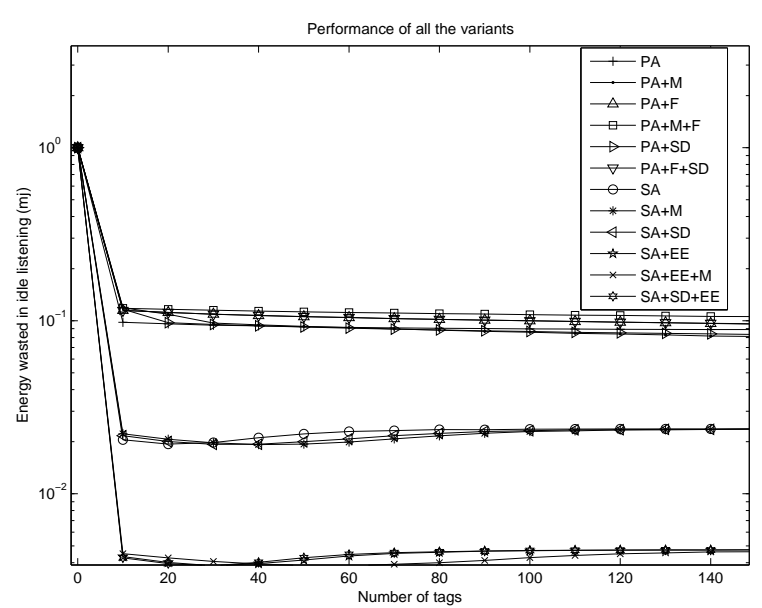

Fig. 15. Energy wasted in idle listening to read $n$ tags by Pure and Slotted Aloha variants.

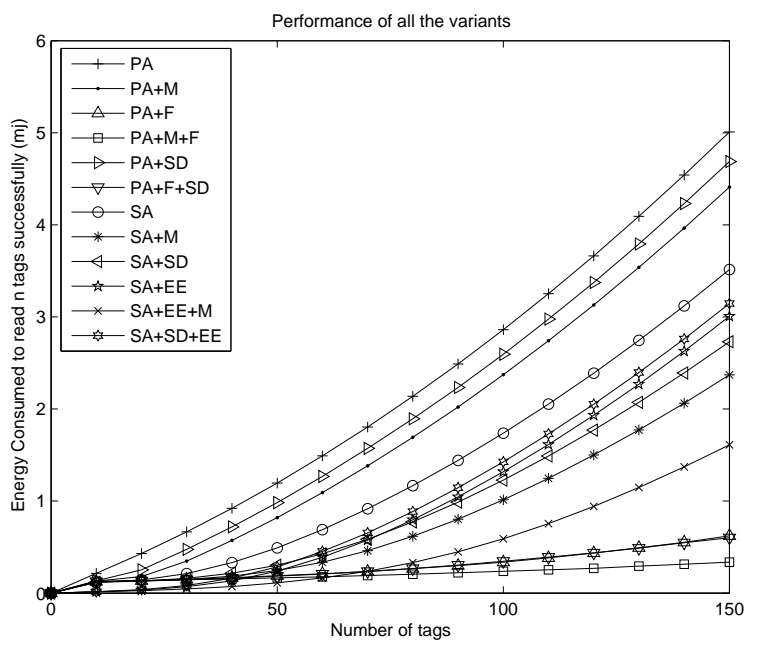

Fig. 16. Energy consumed to read $n$ tags by Pure and Slotted Aloha variants.

\section{CONCLUSION}

This paper has investigated how the battery life-time of the reader is affected by the anti-collision protocol implemented. Our main conclusions are as follow:

- Fast mode variants experience the smallest number of collisions. This means, a reader using these variants will be able to identify the highest number of tags quickly.

- Early end variants reduce energy consumption in idle listening significantly. However, this achievement does not have any impact on the number of collisions.

- Pure Aloha with fast mode and muting is the variant that can read the highest number of tags in a short period of time. Overall, this variant has the smallest number of collisions, and hence the highest read rate.

\section{ACKNOWLEDGMENT}

We acknowledge and thank the support of the Australia Research Council, grant number DP0559769. 


\section{REFERENCES}

[1] F. Bo, L. Jin-Tao, G. Jun-Bo, and D. Zhen-Hua. ID-binary tree stack anti-collision algorithm for RFID. In Proceedings of the 11th IEEE Symposium on Computers and Communications, pages 207-212, PulaCagliari, Sardinia, Italy, 2006.

[2] K. Finkenzeller. RFID Handbook: Fundamentals and Applications in Contactless Smart Cards and Identification. John Wiley and Sons Ltd, 2003.

[3] Intel. Intel sensor nets/RFID research areas. Webpage. http://www.intel.com/research/exploratory /wireless_sensors.htm.

[4] B.-S. Kang, J.-Y. Kim, J. Jwa, and D.-Y. Yang. Bin-slotted hybrid search algorithm for multiple RFID arbitration. In ICWN, pages 164-172, 2006.

[5] D. Klair, K.-W. Chin, R. Raad, and D. Lowe. A spatially aware RFIDenhanced wireless sensor network. In (IEEE PerCom): Google PhD Forum, Hong Kong, China, Mar. 2008.

[6] D. K. Klair, K.-W. Chin, and R. Raad. An investigation into the energy efficiency of pure and slotted Aloha based RFID anti-collision protocols. In IEEE WoWMoM, Helsinki, Finland, Oct. 2007.

[7] D. K. Klair, K.-W. Chin, and R. Raad. On the suitability of framed Aloha based RFID anti-collision protocols for RFID-Enhanced WSNs. In IEEE ICCCN. Honolulu, Hawaii, USA, 2007.

[8] V. Namboodiri and L. Gao. Energy-aware tag anti-collision protocols for RFID systems. In IEEE PerCom, pages 23-46, 2007.

[9] M. Schwartz. Telecommunication Networks Protocols, Modeling and Analysis. Addison-Wesley, USA, 1988.

[10] D. H. Shih, P. L. Sun, D. C. Yen, and S. M. Huang. Taxonomy and survey of RFID anti-collision protocols: Short survey. Computer Communications, 29(11):2150-2166, 2006.

[11] SkyeTek. Skye module M1-Mini. Datasheet. http://www.skyetek.com/Portals//0/SkyeModule_M1Mini_060426.pdf.

[12] The MathWorks. Matlab. http://www.mathworks.com/.

[13] R. Want. An introduction to RFID technology. IEEE Pervasive Computing, 5(1):25-33, 2006.

[14] E. Zurich and L. A. Burdet. RFID multiple access methods. Technical Report, ETH Zurich, 2004. 